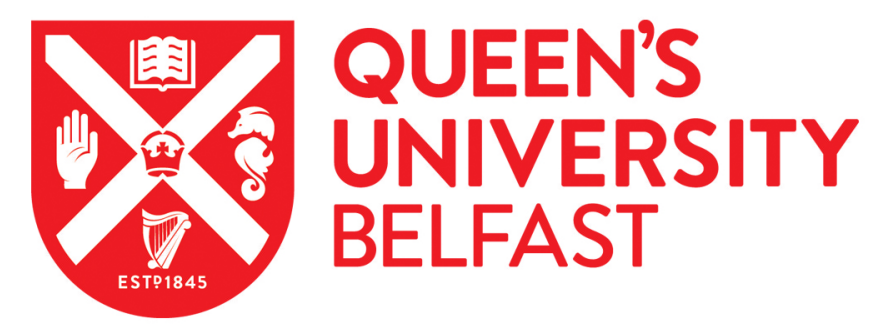

\title{
First Evidence of Enhanced Recombination in Astrophysical Environments and the Implications for Plasma Diagnostics
}

Nemer, A., Sterling, N. C., Raymond, J., Dupree, A. K., García-Rojas, J., Wang, Q., Pindzola, M. S., Ballance, C., \& Loch, S. D. (2019). First Evidence of Enhanced Recombination in Astrophysical Environments and the Implications for Plasma Diagnostics. The Astrophysical Journal Letters, 887(1), [L9]. https://doi.org/10.3847/2041-8213/ab5954

Published in:

The Astrophysical Journal Letters

Document Version:

Publisher's PDF, also known as Version of record

Queen's University Belfast - Research Portal:

Link to publication record in Queen's University Belfast Research Portal

Publisher rights

(c) 2019. The American Astronomical Society. All rights reserved. This work is made available online in accordance with the publisher's policies. Please refer to any applicable terms of use of the publisher.

\section{General rights}

Copyright for the publications made accessible via the Queen's University Belfast Research Portal is retained by the author(s) and / or other copyright owners and it is a condition of accessing these publications that users recognise and abide by the legal requirements associated with these rights.

Take down policy

The Research Portal is Queen's institutional repository that provides access to Queen's research output. Every effort has been made to ensure that content in the Research Portal does not infringe any person's rights, or applicable UK laws. If you discover content in the Research Portal that you believe breaches copyright or violates any law, please contact openaccess@qub.ac.uk. 


\title{
First Evidence of Enhanced Recombination in Astrophysical Environments and the Implications for Plasma Diagnostics
}

\author{
A. Nemer ${ }^{1,2}$ (D) , N. C. Sterling ${ }^{3}$ (D) J. Raymond ${ }^{4}$ (D) A. K. Dupree ${ }^{4}$ (D) J. García-Rojas ${ }^{5,6}$ (D), Qianxia Wang ${ }^{1,7}$, M. S. Pindzola ${ }^{1}$, \\ C. P. Ballance ${ }^{8}$, and S. D. Loch ${ }^{1}$ \\ ${ }^{1}$ Auburn University, Auburn, AL, USA; anemer@princeton.edu \\ ${ }^{2}$ Princeton University, Princeton, NJ, USA \\ ${ }^{3}$ University of West Georgia, Carrollton, GA, USA \\ ${ }^{4}$ Center for Astrophysics|Harvard \& Smithsonian, Cambridge, MA, USA \\ ${ }^{5}$ Instituto de Astrofísica de Canarias, La Laguna, Tenerife, Spain \\ ${ }^{6}$ Universidad de La Laguna, La Laguna, Tenerife, Spain \\ ${ }^{7}$ Rice University, Houston, TX, USA \\ ${ }^{8}$ Queen's University of Belfast, Belfast, UK \\ Received 2019 September 16; revised 2019 November 15; accepted 2019 November 20; published 2019 December 5
}

\begin{abstract}
We report the first unambiguous observational evidence of Rydberg Enhanced Recombination (RER), a potentially important recombination mechanism that has hitherto been unexplored in low-temperature photoionized plasmas. RER shares similarities with dielectronic recombination, with the difference that the electron is captured into a highly excited state below the ionization threshold—rather than above the threshold—of the recombining ion. We predict transitions of carbon and oxygen ions that are formed via the RER process, and their relative strengths with collisional-radiative spectral models. Optical C II RER features are detected in published high-resolution spectra of eight planetary nebulae, and a C III transition has been found in the ultraviolet spectrum in a symbiotic star system. The relative intensities of these lines are consistent with their production by this recombination mechanism. Because RER has not previously been accounted for in photoionized plasmas, its inclusion in models can significantly impact the predicted ionization balance and hence abundance calculations of important astrophysical species. Calculations for $\mathrm{C}^{+}$suggest that the enhancement in the total recombination rate can amount to a factor of 2.2 at $8100 \mathrm{~K}$, increasing to 7.5 at $T=3500 \mathrm{~K}$. These results demonstrate the importance of including RER in models of photoionized astrophysical plasmas and in elemental abundance determinations.
\end{abstract}

Unified Astronomy Thesaurus concepts: Atomic spectroscopy (2099); Planetary nebulae (1249); Symbiotic binary stars (1674); Radiative transfer simulations (1967)

\section{Introduction}

A new recombination process, proposed by Robicheaux et al. (2010), modifies current descriptions of electron-ion recombination in plasmas to include a process that we refer to as Rydberg Enhanced Recombination (RER). This new mechanism may have far-reaching implications, affecting elemental abundance determinations and ionization equilibrium solutions. However, there was no previous evidence for RER in laboratory or astrophysical plasmas. In this Letter we present spectroscopic evidence of RER in planetary nebulae (PNe) and symbiotic stars, and explore its effects on their physical conditions and chemical compositions.

The charge state distribution of elements in astrophysical nebulae results from a balance between photoionization and recombination processes. The main recombination processes in photoionized plasmas are non-resonant, radiative recombination, and resonant dielectronic recombination (DR). DR is often the dominant process and involves a free electron being captured into a doubly excited state (resonance), which then decays to a lower level via photon emission. Robicheaux et al. (2010) introduced RER, which includes resonances just below the ionization threshold that are not considered in DR. RER is analogous to DR, but includes three steps (indicated in Figure 1): (1) low-energy free electrons recombine to high- $n$ singly excited states of an ion (called Rydberg states), (2) radiationless transition to a resonance below the threshold (which we call dielectronic auto-transfer), (3) radiative transitions to lower levels to complete the process.

Photoionized nebulae are ubiquitous, diagnostically important objects (Peimbert et al. 2017), and have contributed significantly to our knowledge of the chemical evolution of the cosmos. These plasmas are ionized by low-mass dying stars in the case of PNe, a hot compact object in symbiotic binaries, or young, massive stars in $\mathrm{H}$ II regions. Accurate elemental abundance determinations are essential in benchmarking existing models of stellar evolution, nucleosynthesis, galactic composition and kinematics, and cosmology (Zaritsky et al. 1994; Hamann \& Ferland 1999; Savin 2000; Henry et al. 2018). However, plasma diagnostics suffer from various issues, including discrepancies in temperature and abundance determinations (Peimbert et al. 2017). Ferland et al. (1998) showed that the lack of reliable DR rates is the dominant uncertainty in ionization balance calculations of photoionized plasmas. Additionally, ionization correction factors are used to convert ionic abundances into elemental abundances (Delgado-Inglada et al. 2014). Such corrections for unobserved ions depend on accurate ionization and recombination rate coefficients. Neglecting the contribution of RER to recombination thus leads to inaccurate abundance determinations. This will affect abundance ratios such as those used to constrain the intergalactic medium (Savin 2000) and nucleosynthesis models (Henry et al. 2018).

The physical conditions of low-temperature $(\sim 10,000 \mathrm{~K})$ photoionized nebulae make them ideal "astrophysical 


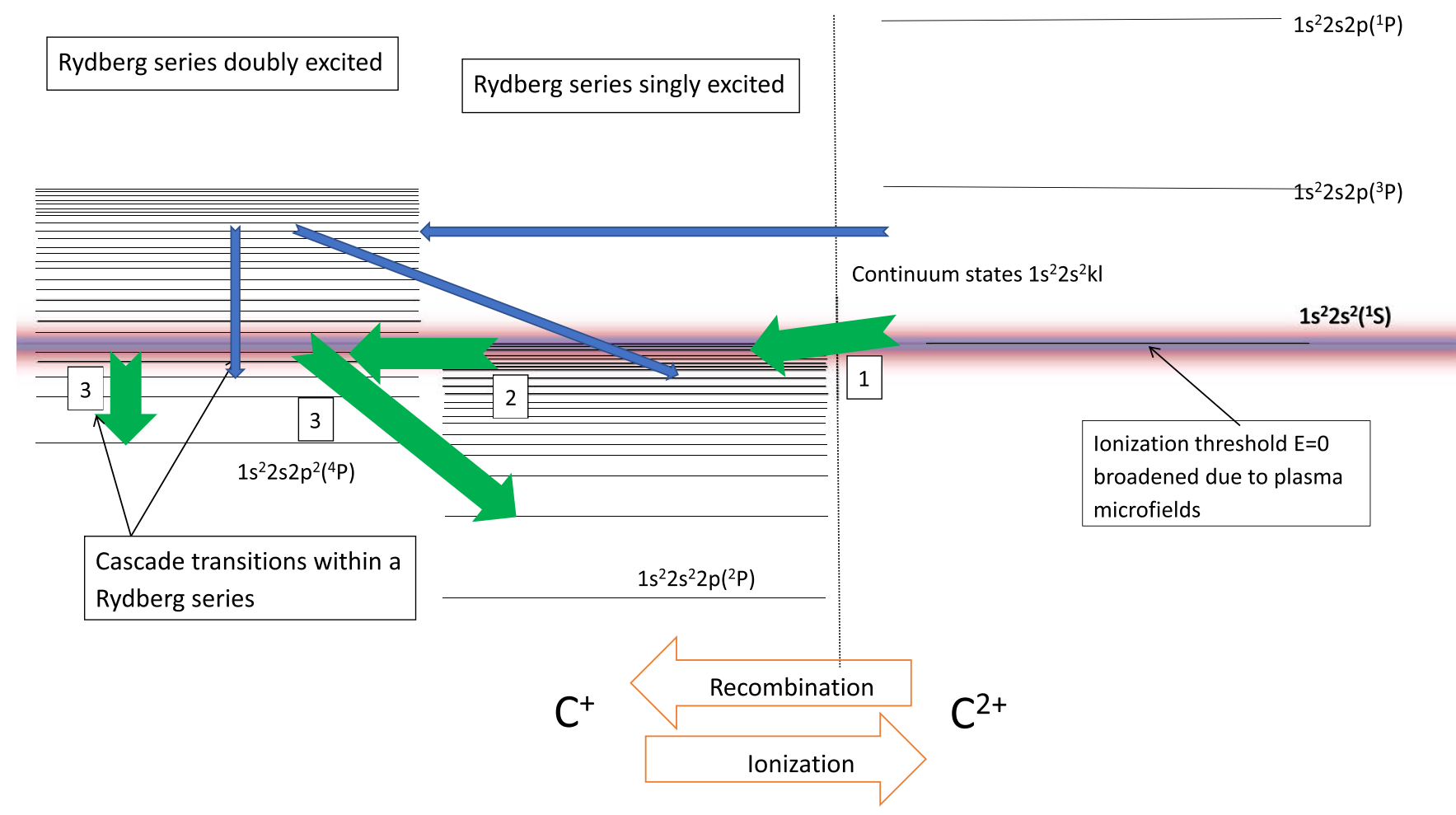

Figure 1. Energy level diagram. The right hand-side shows $\mathrm{C}^{2+}$ recombining into $\mathrm{C}^{+}$(left side). The blurred line represents the ionization threshold $\left(\mathrm{C}^{2+}\right.$ ground state). Two series of energy levels are shown, the singly excited $1 \mathrm{~s}^{2} 2 \mathrm{~s}^{2} \mathrm{nl}$ series, and the doubly excited $1 \mathrm{~s}^{2} 2 \mathrm{~s} 2 \mathrm{pnl}$ series. The thin blue arrows trace DR, and the thick green arrows denote RER, with numbered steps defined in the text.

laboratories" to search for emission lines produced by RER, given the low electron temperatures needed for RER. In this Letter, we identify ions that could be affected by RER, and present spectroscopic evidence of RER using high-resolution optical spectra of PNe and ultraviolet (UV) spectra of a symbiotic binary.

\section{Background Theory}

Figure 1 illustrates the DR and RER processes. Historically, DR has been assumed to be initiated only from free electron states in the continuum (blue arrows in Figure 1). Robicheaux et al. (2010) indicated that a similar process, RER, is possible between Rydberg states and below-threshold resonances of the same ion (Figure 1, green arrows). Such transitions have been observed in charge exchange experiments (Ali et al. 2016). Quantum mechanically this is a result of wavefunction mixing, with the ion in a superposition of each of the states. Because RER involves low-energy electron capture into Rydberg states, this process is most effective in low-temperature plasmas.

To test for the occurrence of RER in astrophysical nebulae, it is necessary to predict spectral lines produced by this mechanism. Quantifying the effect of RER on the ionization equilibrium additionally requires the re-evaluation of total recombination rate coefficients. To address these needs, two multi-configuration atomic structure codes were modified, the Breit-Pauli code AUTOSTRUCTURE (Badnell 2011) and the Dirac-Fock code GRASP (Dyall et al. 1989).

As shown by Robicheaux et al. (2010), calculating RER rates is analogous to the DR methodology. DR cross sections are converted to plasma rate coefficients via integration over a Maxwellian distribution of free electrons. In contrast, RER involves Rydberg states, and the rate coefficients are computed by integrating over their population distributions. The capture rate from the Rydberg states into a resonance $(j)$ below the ionization threshold is

$$
\alpha_{j}^{\text {below }}\left(T_{e}\right)=\left(\frac{4 \pi a_{o}^{2} I_{H}}{k_{B} T_{e}}\right)^{3 / 2} \frac{\omega_{j}}{\omega_{+}} e^{-E_{c} / k_{B} T_{e}} A_{j, E_{c}}^{a} b_{j}
$$

where $I_{H}$ is the Rydberg constant, $k_{B}$ is Boltzmann's constant, $T_{e}$ is the plasma electron temperature, $\omega_{j}$ and $\omega_{+}$are the statistical weights of the doubly excited state and ground state of the recombining ion, respectively, $E_{c}$ is the energy of the resonance relative to the ionization potential, $A^{a}$ is the dielectronic auto-transfer rate to Rydberg states, and $b_{j}$ is the departure factor (described below). To obtain the enhancement to the total recombination rate for that charge state, the rate coefficient is multiplied by a radiative branching ratio and summed over all below-threshold states $j$ that can mix with the Rydberg states.

Resonances below the ionization potential are therefore populated by RER (and other processes discussed later), and depopulated by radiative decay $\left(A^{r}\right)$ and dielectronic autotransfer to Rydberg states $\left(A_{a}\right)$. Thus the population of the doubly excited state is

$$
N_{j}=\frac{N^{+} n_{e} \alpha_{j}^{\mathrm{RER}}+N^{+} n_{e} \alpha_{j}^{\text {cascade }}+N^{g} n_{e} q_{j}^{\text {fluorescence }}}{\sum_{k} A_{r}+\sum_{l} A_{a}},
$$

where $N^{+}$and $N^{g}$ are the populations of the ground states of the recombining and recombined ions, and $\alpha_{j}$ and $q_{j}$ represent the effective rates to populate the resonance. This population density is multiplied by spontaneous emission rates to evaluate 
Table 1

$\mathrm{C}$ and $\mathrm{O}$ Transitions Arising from RER

\begin{tabular}{|c|c|c|c|}
\hline Ion & Term (multiplet) & $\begin{array}{l}\text { Energy Below } \\
\text { Ionization Poten- } \\
\text { tial }(\mathrm{eV})\end{array}$ & $\begin{array}{l}\text { Wavelengths of } \\
\text { Strongest Transi- } \\
\text { tions }^{\text {a }}(\AA)\end{array}$ \\
\hline C II & $1 \mathrm{~s}^{2} 2 \mathrm{~s} 2 \mathrm{p}\left({ }^{3} \mathrm{P}^{\circ}\right) 3 \mathrm{~d}\left({ }^{4} \mathrm{D}\right)$ & $0.010097-0.011355$ & $651.21,651.39$ \\
\hline $\mathrm{C}$ II & $1 \mathrm{~s}^{2} 2 \mathrm{~s} 2 \mathrm{p}\left({ }^{3} \mathrm{P}^{\circ}\right) 3 \mathrm{~d}\left({ }^{4} \mathrm{~F}\right)$ & $0.105355-0.112927$ & $\begin{array}{l}\mathbf{7 1 1 5 . 5 3}, 7134.03 \\
\mathbf{7 1 1 2 . 9 4}, \mathbf{7 1 1 9 . 7 3}\end{array}$ \\
\hline C III & $1 \mathrm{~s}^{2} 2 \mathrm{p} 4 \mathrm{p}\left({ }^{1} \mathrm{D}\right)$ & $0.07475-0.05254$ & $\begin{array}{c}1581.43,416.77 \\
1623.25,1512.93\end{array}$ \\
\hline C III & $1 \mathrm{~s}^{2} 2 \mathrm{p} 4 \mathrm{p}\left({ }^{3} \mathrm{~F}\right)$ & 0.05148 & $\begin{array}{l}1593.65,1594.27 \\
2799.19, \mathbf{1 5 5 3 . 3 8}\end{array}$ \\
\hline O III & $\begin{array}{c}1 \mathrm{~s}^{2} 2 \mathrm{~s}^{2} 2 \mathrm{p}^{2}\left({ }^{1} \mathrm{~S}\right) 4 \mathrm{~s} \\
\left({ }^{2} \mathrm{~S}\right)\end{array}$ & 0.130312 & 413.65 \\
\hline O III & $\begin{array}{c}1 \mathrm{~s}^{2} 2 \mathrm{~s}^{2} 2 \mathrm{p}^{2}\left({ }^{1} \mathrm{D}\right) 5 \mathrm{~s} \\
\left({ }^{2} \mathrm{D}\right)\end{array}$ & 0.635618 & $397.9,420.7$ \\
\hline O III & $\begin{array}{c}1 s^{2} 2 s^{2} 2 p^{2}\left({ }^{4} P\right) 4 p \\
\left({ }^{3} \mathrm{D}\right)\end{array}$ & 0.04554 & $225.9,671.8$ \\
\hline O III & $\begin{array}{c}1 \mathrm{~s}^{2} 2 \mathrm{~s}^{2} 2 \mathrm{p}^{2}\left({ }^{4} \mathrm{P}\right) 4 \mathrm{p} \\
\left.{ }^{5} \mathrm{P}\right)\end{array}$ & $0.45183-0.59911$ & $\begin{array}{c}2430.3,2427.66 \\
2426.78\end{array}$ \\
\hline
\end{tabular}

Note.

${ }^{a}$ Wavelengths shorter than $2000 \AA$ are in vacuum, and wavelengths above 2000 are in air. Observed lines are highlighted in bold.

the emission per unit volume per second for the belowthreshold transitions.

We utilized collisional-radiative theory, with a modified version of the ADAS code (Summers et al. 2006), to determine the Rydberg populations. ADAS204 was used as it includes all collisional and radiative populating processes for the Rydberg states (dominated by three-body and radiative recombination) and depopulating processes (mainly collisional and photoionization), then solves the quasi-static equilibrium equations to produce level populations. Rydberg populations are described in terms of a departure coefficient, defined as the ratio between the level populations and their local thermal equilibrium (LTE) value. The highest n-shells are close to LTE, and below about $n=200$ the populations fall below the LTE value due to less frequent collisions and faster radiative decay.

A spectral synthesis code was developed that uses these atomic data to model the intensities of the RER spectral lines, and accurate energies (NIST database; Reader et al. 2012) to predict the wavelengths.

\section{Results}

\subsection{Predicted RER Emission Lines}

We established criteria to identify transitions whose upper levels are likely populated by RER and thus may produce detectable emission lines. The doubly excited upper levels must (i) be propinquitous to the ionization threshold, (ii) mix with Rydberg states, and (iii) be from an ion widely observed in astrophysical nebulae. We investigated the first two rows of the periodic table for transitions that fit these criteria, and C II, C III, $\mathrm{O}$ II, and $\mathrm{O}$ III were the most promising, with accurate energy levels available for the resonances. Table 1 shows the candidates for the search.

Energies from the NIST database were used for the wavelength predictions. We focused on the $\mathrm{C}$ II lines $\lambda 7112.94,7119.73$ and 7115.53 due to the availability of high-resolution optical spectra of PNe, and C III $\lambda 1553.38$ using high-resolution UV spectra of symbiotic binaries. The
UV RER lines are generally predicted to be stronger than optical lines due to higher dielectronic auto-transfer rates and branching ratios.

\subsection{Spectroscopic Evidence for RER}

High-resolution spectroscopy is critical for identifying RER lines, both to resolve these lines from nearby features of other species and to provide greater line-to-continuum contrast for weak features. We searched for optical C II RER lines (Table 1) in published high-resolution spectra of seven $\mathrm{PNe}$ observed with 4-8 m telescopes: IC 418 (Sharpee et al. 2003); IC 2501, IC 4191, NGC 2440, NGC 7027 (Sharpee et al. 2006); NGC 6369 (García-Rojas et al. 2012); and NGC 3918 (GarcíaRojas 2015). We also studied an unpublished spectrum of $\mathrm{Hb}$ 12 , obtained with the $2 \mathrm{D}$ coudé spectrograph on the $2.7 \mathrm{~m}$ Harlan J. Smith Telescope at McDonald Observatory (Sherrard et al. 2017). We show six of these objects that exhibit C II RER lines (Figure 2(a)), whose intensities relative to $\mathrm{H} \beta$ are given in Table 2.

To verify the identities of $\mathrm{C}$ II $\lambda \lambda 7112.94,7119.73$ and 7115.53, we utilize the Atomic Line List v2.05b2 ${ }^{9}$ to search for other possible identifications with rest wavelengths within $1 \AA$. We considered forbidden transitions of atomic ions with excitation energies below $10 \mathrm{eV}$, and permitted transitions of elements in the first three rows of the periodic table. For potential alternative identifications, we searched for multiplet members, lines from the same upper level, and forbidden transitions of iron-peak elements, the strongest optical lines expected given the physical conditions of the nebulae. Using this method, we ruled out alternative identifications for these C II lines, in agreement with the identifications of Storey \& Sochi (2013). Based on our vetting criteria and the detection of multiplet members, we are confident that C II $\lambda \lambda 7112.94$, 7119.73 and 7115.53 have been detected in each of the eight $\mathrm{PNe}$ that we investigated.

It is also important to confirm that the C II RER lines are not produced by another mechanism. Fluorescence is quantummechanically forbidden to populate these resonances from the ground (or metastable) states. Alternatively, cascade from higher (i.e., above threshold, populated by DR) doubly excited states in the same Rydberg series (e.g., $1 \mathrm{~s}^{2} 2 \mathrm{~s} 2 \mathrm{pnl}$ ) can provide a populating mechanism. We searched the spectra for cascade lines that can populate the upper level of C II $\lambda 7115.53$ based on their branching ratios, namely C II 3876 and $5259 \AA$ (Table 2). In five objects (NGC 6369, NGC 7027, NGC 2440, NGC 3918, and $\mathrm{Hb}$ 12), the cascade lines were either not detected, or are too weak to explain the observed intensities of the RER lines. This indicates that the $\mathrm{C}$ II multiplet $\lambda \lambda 7112.94$, 7115.53 , and 7119.73 is populated almost entirely by RER. In two PNe (IC 418 and IC 2501), the cascade contribution was close to the observed intensity in the two lines, indicating that RER has a negligible contribution. In IC 4191, the cascade line intensities are much larger than predicted compared to that of $\lambda 7115.53$, suggesting that $\mathrm{C}$ II $\lambda \lambda 3876$ and 5259 are either blended with unknown features or misidentified. When the summed cascade portion of the C II $\lambda 7115.53$ intensity is larger than that observed, we give a negative factor in Table 2.

For a quantitative comparison of observed and predicted line intensities, it is necessary to consider both observational and theoretical uncertainties. The emission lines produced by RER

\footnotetext{
9 http://www.pa.uky.edu/ peter/newpage/
} 

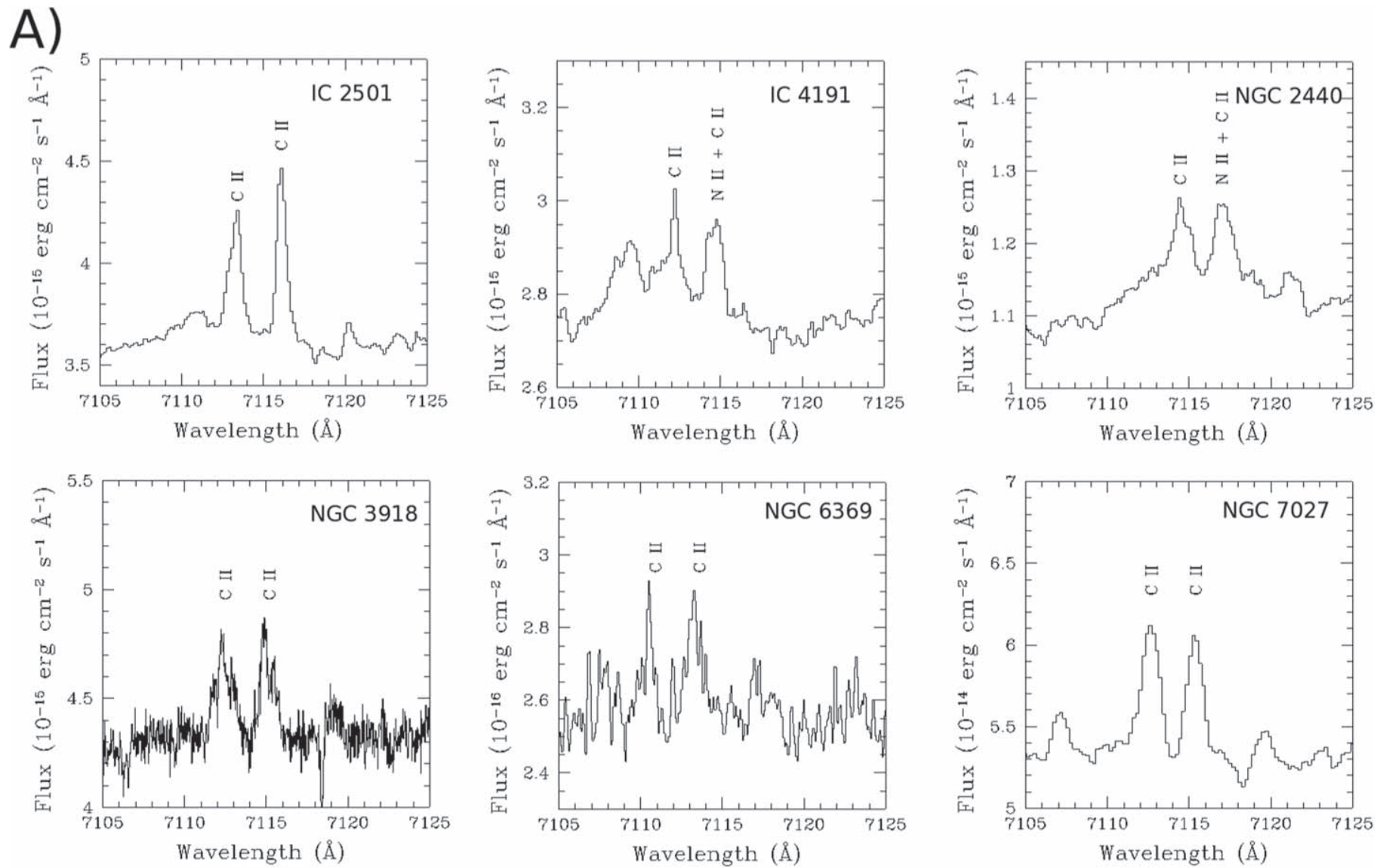

B)

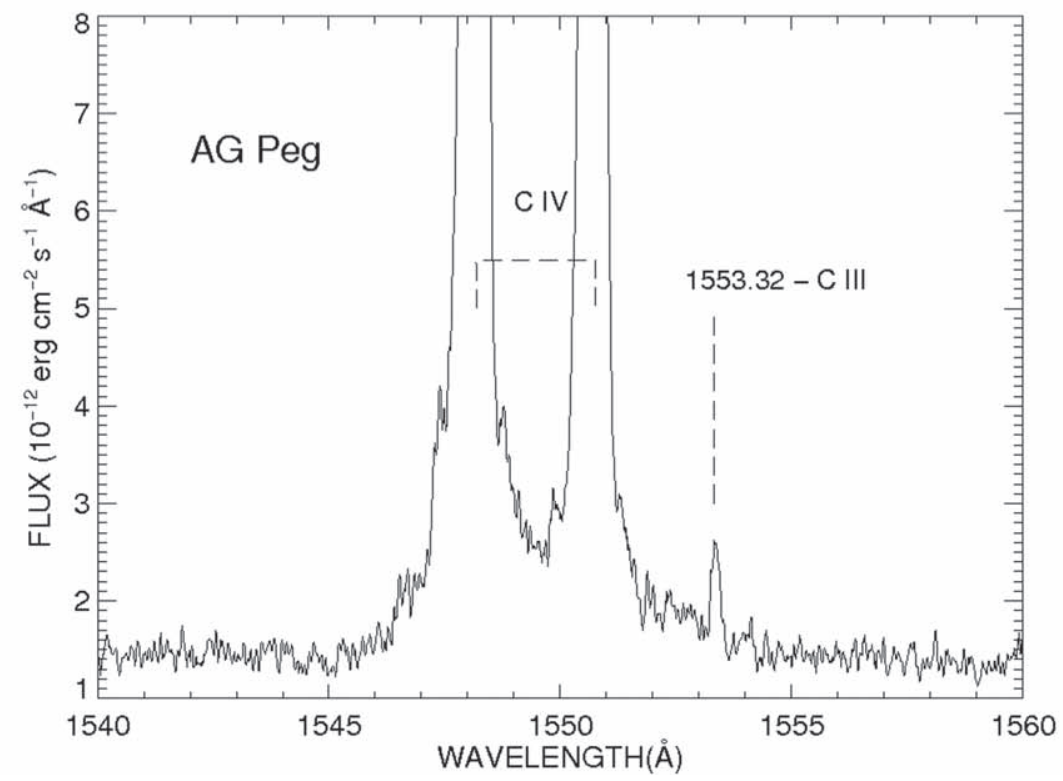

Figure 2. A: high-resolution spectra showing the C II $\lambda \lambda 7112.94$ and 7115.53 RER lines in the PNe IC 2501, IC 4191, NGC 2440, NGC 7027, NGC 3918, and NGC 6369. B: C III $\lambda 1553.32$ in the Hubble Space Telescope (HST)/Goddard High-Resolution Spectrograph (GHRS) z27e020at spectrum (program 5360, PI: Nussbaumer) of AG Pegasi.

are weak, and the error bars on their observed intensities are $20 \%-25 \%$ in the objects investigated. Moreover, flux calibration of cross-dispersed echelle spectra can lead to additional systematic uncertainties. From the atomic physics perspective, the dielectronic auto-transfer rates are uncertain by $\sim 30 \%$, depending on the coupling model adopted to compute them. The largest uncertainty in our predicted RER line intensities and modified recombination rate coefficients is likely in the computed Rydberg populations. These were calculated from collisional-radiative models that require accurate knowledge of 
Table 2

Intensities (on the Scale $I\left(\mathrm{H}_{\beta}=100\right)$ ) of the $\mathrm{C}$ II RER Line $\lambda 7115.53$ and Cascade Lines $\lambda 3876$ and 5259 that Compete with RER to Populate these Levels

\begin{tabular}{|c|c|c|c|c|c|}
\hline PN & $10^{-3} I(7115) / I\left(\mathrm{H}_{\beta}\right)$ & $10^{-3} I(3876) / I\left(\mathrm{H}_{\beta}\right)$ & $10^{-3} I(5259) / I\left(\mathrm{H}_{\beta}\right)$ & $\begin{array}{c}\text { RER } \\
\text { Contribution } \\
\text { to } I(7115)^{\mathrm{a}}\end{array}$ & $\begin{array}{l}I(7112) / \\
I(7115)^{\mathrm{b}}\end{array}$ \\
\hline $\mathrm{Hb} 12$ & $4.48 \pm 0.90$ & $\ldots$ & $\ldots$ & 1.00 & $1.76 \pm 0.50$ \\
\hline IC 2501 & $9.80 \pm 2.94$ & $14.00 \pm 1.50$ & $5.00 \pm 1.00$ & 0.22 & $0.76 \pm 0.32$ \\
\hline IC 418 & $4.30 \pm 0.43$ & $6.90 \pm 1.38$ & $6.33 \pm 1.26$ & -0.34 & $1.21 \pm 0.17$ \\
\hline IC 4191 & $3.10 \pm 0.62$ & $96.00 \pm 10.10$ & $\ldots$ & -10.00 & $0.90 \pm 0.25$ \\
\hline NGC 2440 & $23.00 \pm 4.60$ & $\ldots$ & $3.60 \pm 0.72$ & 0.92 & $1.13 \pm 0.32$ \\
\hline NGC 3918 & $6.80 \pm 1.36$ & $\ldots$ & $\ldots$ & 1.00 & $1.04 \pm 0.29$ \\
\hline NGC 6369 & $11.00 \pm 4.40$ & $\cdots$ & $\ldots$ & 1.00 & $0.636 \pm 0.36$ \\
\hline NGC 7027 & $14.70 \pm 1.47$ & $\cdots$ & $7.90 \pm 1.58$ & 0.72 & $1.22 \pm 0.17$ \\
\hline
\end{tabular}

Notes.

a See the text for discussion of negative numbers.

b The last column shows the observed ratio of the strongest C II RER multiplet lines.

the plasma conditions (e.g., temperature and density) of the emitting region(s), and comprehensive inclusion of RER. Our model predictions assumed uniform electron temperature and density, thus neglecting local variations of these parameters (Liu et al. 2000).

In Table 2 we show the observed intensity ratios of $\mathrm{C}$ II 7112.94 and $7115.53 \AA$, which range between 0.76 and 1.78 (with an average of 1.15). These values agree with the predicted ratio of 0.63 to within the observational and modeling uncertainties estimated to be $40 \%$.

Because RER transitions in the UV are predicted to be stronger than their optical counterparts, we investigated archival UV spectra of PNe and symbiotic binaries. Of the PNe whose optical spectra we investigated, sufficiently highresolution IUE spectra exist for $\mathrm{Hb} 12$, IC 418, NGC 3918, and NGC 7027. The individual data sets do not exhibit RER lines, but the low signal-to-noise ratio of the spectra do not provide strong constraints.

Symbiotic stars are binary systems in which a white dwarf accretes gas from a red giant. We analyzed the UV spectrum of the AG Pegasi symbiotic, whose luminosity decreased by a factor of four between 1984 and 1994 (Eriksson et al. 2006), as the broad C IV resonance lines from the white dwarf wind declined. The C III $\lambda 1553.8$ RER line was not visible in the earlier spectrum, but became apparent after the C IV emission faded (Figure 2(b)). The HST/GHRS observations used by Eriksson et al. (2006) clearly revealed the $1553 \AA$ line, but it was not identified by those authors. We verified the identity of this feature in a manner similar to that for the optical C II lines, comparing against the line lists in Eriksson et al. (2006), and visual inspection of the IUE SWP47715 spectrum (program PA047, PI: Vogel). Due to the preponderance of fluorescently excited Fe and Co transitions in the UV spectrum of AG Peg, we expanded our search to include permitted lines from elements up to $\mathrm{Zn}$, but found no plausible alternate identifications.

In summary, we identify C II multiplet $\lambda \lambda 7112.94,7115.53$, and 7119.73 lines in eight PNe, and show that RER is the dominant populating mechanism for the upper levels of these transitions in five objects. The RER line C III $1553.38 \AA$ is seen in the AG Pegasi symbiotic binary. These observations and our analysis represent the first empirical evidence of the RER mechanism.

\section{Implications of RER for Photoionized Plasmas}

\subsection{Effects of RER on Abundance Determinations and the Ionization Balance of Nebulae}

The inclusion of RER in the analysis of astrophysical plasmas is expected to change the ionization equilibrium, due to an increased rate of recombination, and hence elemental abundances.

To demonstrate these effects, we modified the DR rate coefficients in Cloudy (Ferland et al. 2017) to include contributions from RER. Figure 3 illustrates the modified recombination rate coefficients for $\mathrm{C}^{2+}$, comparing those used in Cloudy (Colgan et al. 2003) to our calculations and to measured rates (Fogle et al. 2005); the recommended rates are a hybrid of measured and calculated values. Below $\sim 10,000 \mathrm{~K}$ recombination rates are dominated by below-threshold resonances, and RER produces rates that are substantially higher than other recombination processes. The enhancement to the recombination rates due to RER can be seen from the difference between the measured rates (red) and the recommended rates (purple), a factor of 2.2 at $T=8100 \mathrm{~K}$, and 7.5 at $T=3500 \mathrm{~K}$.

In our models, RER only modifies the populations of the upper levels of transitions in Table 1 (and hence their emissivities), but RER should be included in a comprehensive collisional-radiative modeling to predict its effects on the populations of all excited states (and hence their emission spectra). $\mathrm{C}^{2+}$ abundances are often deduced from optical lines like C II $\lambda 4267$, for which the effect of RER is yet to be determined. To illustrate how RER can affect line ratios (and hence abundance determinations), we calculated the line ratio of C II $\lambda 4267$ to C II $\lambda 7115$ with and without RER as a function of temperature. We found that the ratio is reduced by two orders of magnitude at $T=5000 \mathrm{~K}$, and by an order of magnitude at $T=10,000 \mathrm{~K}$ due to the enhancement of $\mathrm{C} \mathrm{II}$ $\lambda 7115$ line emissivity. The effect on other $\mathrm{C}$ II lines requires full inclusion of RER in emissivity calculations, and incorporating density and temperature inhomogeneities (Liu et al. 2000) in radiative transfer models.

We evaluated models for the "Paris Meeting" PN, H II region, and AGN narrow line region simulations in the Cloudy test suite ${ }^{10}$ both with and without the modified recombination rate coefficients for $\mathrm{C}^{2+}$ and $\mathrm{C}^{3+}$. In the $\mathrm{PN}$ model, we

${ }^{10}$ see www.nublado.org. 

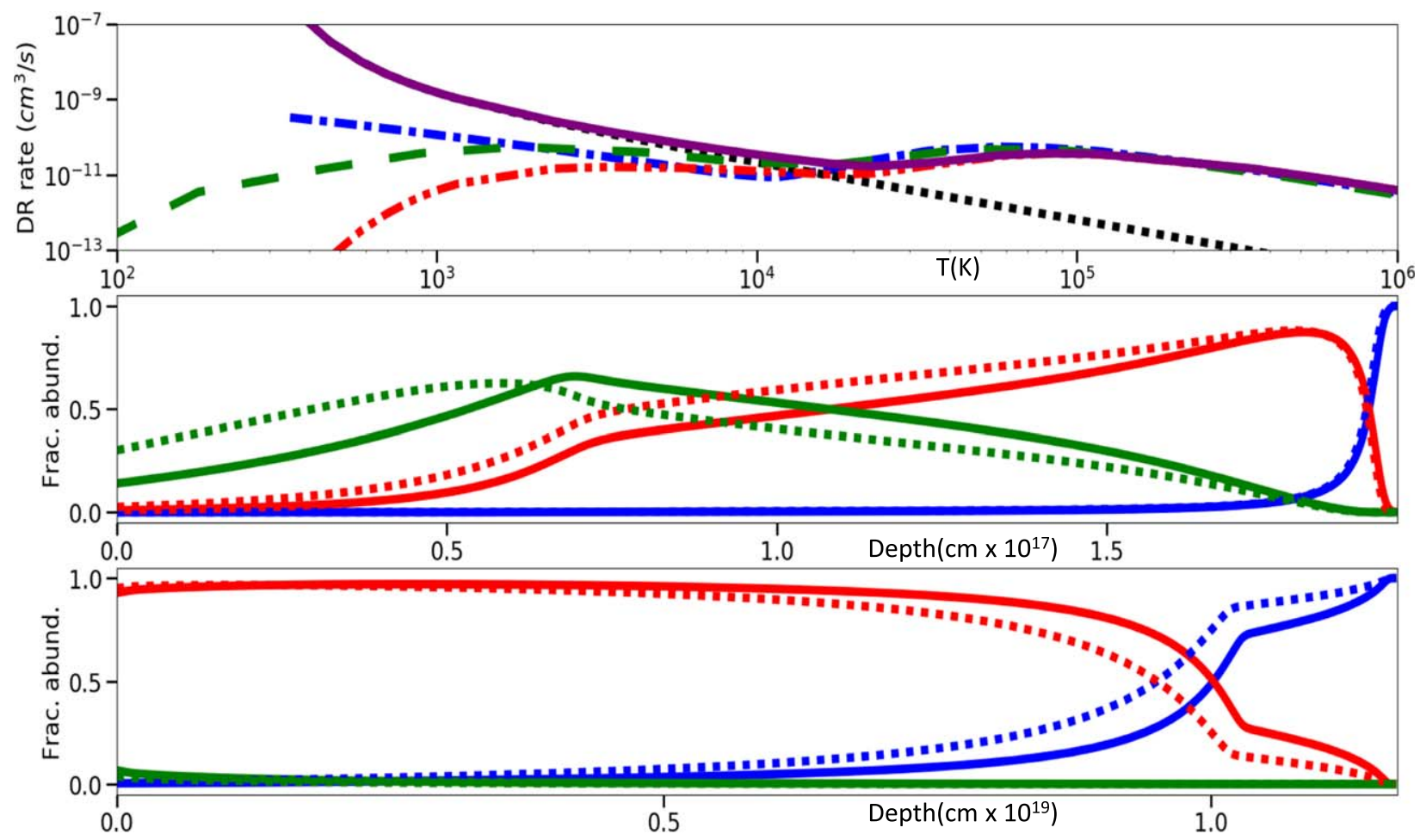

Figure 3. Top panel: comparison among $\mathrm{C}^{2+} \mathrm{DR}$ rates from the literature. The blue (dashed-dotted) curve represents Cloudy rates. The green (dashed) curve is our calculated DR rates, the red (dashed-dotted-dotted) curve is measured DR rates, and the black (dotted) curve is our calculated RER rates. The purple (solid) curve is the recommended total rates. Note the substantial enhancement at $T<10^{4} \mathrm{~K}$ which will decrease the fraction of $\mathrm{C}^{2+}$ ions. Middle panel: fractional abundances of carbon ions as a function of depth in the PN test suite (dotted with old rates and solid with new rates): the blue curve is $\mathrm{C}^{+}$, red is $\mathrm{C}^{2+}$ and the green curve is $\mathrm{C}^{3+}$. Bottom panel: the same as the middle panel, for the H II region Paris model.

introduced density fluctuations spanning $10^{3}-10^{4} \mathrm{~cm}^{-3}$. There is a notable shift in the fractional abundance curves of $\mathrm{C}^{2+}$ and $\mathrm{C}^{3+}$ as a function of radius in each model when enhanced recombination is included (middle and bottom panels of Figure 3). The volume-averaged abundances differ as well, with the $\mathrm{C}^{+}$ionic fraction increased by a factor of $1.15-1.45$ when RER is taken into account, while $\mathrm{C}^{2+}$ decreases by factors of $1.07-1.16$ and $\mathrm{C}^{3+}$ by $1.15-1.50$, depending on the model.

These results demonstrate that including RER in models alters the ionization balance and hence can affect elemental abundance determinations, particularly when ionization correction factors are invoked. The modifications to ionic fractions and elemental abundances could be more pronounced when RER is included for species beyond the two $\mathrm{C}$ ions we considered.

\subsection{Implications of RER for Radio Astronomy}

Dielectronic auto-transfer represents a transition between resonant Rydberg states and doubly excited states, and thus will disturb select Rydberg populations. Collisions with neighboring Rydberg ions can return Rydberg populations to their LTE values, but our models show that dielectronic auto-transfer timescales $(\sim \mathrm{ns})$ are much faster than Rydberg collisional timescales $(\sim 100 \mathrm{~s})$.

Rydberg emission lines from $\mathrm{H}, \mathrm{He}$, and $\mathrm{C}$ have been detected and used to diagnose the physical conditions of nebulae (Gordon \& Sorochenko 2009). In the presence of a strong source of radio continuum emission, the lines are described by non-LTE conditions and can be affected by stimulated emission (Shaver et al. 1977). Lines emission is observed in low density $\left(\sim 10^{4} \mathrm{~cm}^{-3}\right)$, low-temperature $(\leqslant 10,000 \mathrm{~K})$ gas, if there is a mechanism to drive the Rydberg states out of their LTE conditions (Shaver 1980; Anantharamaiah et al. 1993). Dielectronic auto-transfer is a mechanism that can cause population inversions in Rydberg states which leads to stimulated emission in their radio recombination lines (Goldberg 1966). Our model for $\mathrm{C}^{+}$and $\mathrm{C}^{2+}$ indicates that the Rydberg states affected by RER will produce radio emission in the range $0.112 \mathrm{~mm}-4.41 \mathrm{~mm}$ and $2.92 \mathrm{~mm}-5.14 \mathrm{~mm}$, respectively, but further observations are needed to verify this effect.

\section{Conclusions}

The first empirical evidence has been presented for RER in photoionized plasmas. We predict the wavelengths and relative intensities of C II, C III, O II, and O III lines whose upper levels are populated by RER. In existing high-resolution spectra, we identify C II RER lines in the visible spectra of eight PNe, and a C III RER line in the UV spectrum of a symbiotic star. The relative strengths of the $\mathrm{C}$ II lines agree with predictions of our collisional-radiative models within the uncertainties. By adjusting the recombination rate coefficients used in Cloudy to include RER contributions, we demonstrate that the impact of RER on the ionization balance and abundance determinations of astrophysical nebulae is significant. More detailed models that account for small-scale temperature and density fluctuations and include RER contributions for additional ions are needed to study the extent of the implications for RER on elemental abundances. Moreover, our models indicate that RER is most prevalent at low electron temperatures, 
dominating other recombination mechanisms and more drastically altering the charge state distribution. Further modeling and observational efforts are needed to explore these effects.

This work was funded by NASA grant NNZ16AE97G. J.G.R. acknowledges support from the Severo Ochoa excellence program (SEV-2015-0548) and from the State Research Agency (AEI) of the Spanish Ministry of Science, Innovation and Universities (MCIU) and the European Regional Development Fund (FEDER) under grant AYA2017-83383-P. We thank Dr. Francis Robicheaux for his pioneering work in this area.

\section{ORCID iDs}

A. Nemer (ib https://orcid.org/0000-0002-9220-0039

N. C. Sterling (i) https://orcid.org/0000-0002-9604-1434

J. Raymond (i) https://orcid.org/0000-0002-7868-1622

A. K. Dupree (iD https://orcid.org/0000-0002-8985-8489

J. García-Rojas (iD https://orcid.org/0000-0002-6138-1869

\section{References}

Ali, R., Beiersdorfer, P., Harris, C. L., et al. 2016, PhRvA, 93, 012711 Anantharamaiah, K. R., Zhao, J.-H., Goss, W. M., et al. 1993, ApJ, 419, 585 Badnell, N. 2011, CoPhC, 182, 1528
Colgan, J., Pindzola, M. S., Whiteford, A. D., et al. 2003, A\&A, 412, 597 Delgado-Inglada, G., Morisset, C., \& Stasińska, G. 2014, MNRAS, 440, 536 Dyall, K., Grant, I., Johnson, C., et al. 1989, CoPhC, 55, 425 Eriksson, M., Johansson, S., \& Wahlgren, G. M. 2006, A\&A, 175, 157 Ferland, G., Korista, K., Verner, D., et al. 1998, PASP, 110, 761 Ferland, G. J., Chatzikos, M., Guzmán, F., et al. 2017, RMxAA, 53, 385 Fogle, M., Badnell, N., Glans, P., et al. 2005, A\&A, 442, 757 García-Rojas, J., Madonna, S., Luridiana, V., et al. 2015, MNRAS, 452, 2606 García-Rojas, J., Peña, M., Morisset, C., et al. 2012, A\&A, 538, 54 Goldberg, L. 1966, ApJ, 144, 1225

Gordon, M. A., \& Sorochenko, R. L. 2009, Radio Recombination Lines: their Physics and Astronomical Applications (Berlin: Springer)

Hamann, F., \& Ferland, G. 1999, ARA\&A, 37, 487

Henry, R. B. C., Stephenson, B. G., Bertolami, Miller., et al. 2018, MNRAS, 473,241

Liu, X.-W., Storey, P. J., Barlow, M. J., et al. 2000, MNRAS, 312, 585

Peimbert, M., Peimbert, A., \& Delgado-Inglada, G. 2017, PASP, 129, 082001 Reader, J., Kramida, A., \& Ralchenko, Y. 2012, AAS Meeting, 219, 443.01 Robicheaux, F., Loch, S., Pindzola, M., et al. 2010, PhRvL, 105, 233201 Savin, D. W. 2000, ApJ, 533, 106

Sharpee, B., Williams, R., Baldwin, J. A., et al. 2003, ApJS, 149, 157 Sharpee, B., Zhang, Y., Williams, R., et al. 2006, ApJ, 659, 1265 Shaver, P. A. 1980, A\&A, 90, 34

Shaver, P. A., Churchwell, E., \& Rots, A. H. 1977, A\&A, 55, 435

Sherrard, C. G., Sterling, N. C., Dinerstein, H., et al. 2017, AAS meeting, 230, 318.11

Storey, P. J., \& Sochi, T. 2013, MNRAS, 430, 599

Summers, H. P., Dickson, W. J., O'Mullane, M. G., et al. 2006, PPCF, 48, 263 Zaritsky, D., Kennicutt, R. C. J., \& Huchra, J. P. 1994, ApJ, 420, 87 